\title{
Theoretical and Numerical Calculations for the Dynamics of Dilute Colloidal Suspensions of Molecular Particles inside Mesopores
}

\author{
A. Hijazi, A. Atwi, and A. Khater
}

\begin{abstract}
Numerical simulations are developed and applied to analyze the dynamics of dilute colloidal suspensions of molecular nano-particles in dilute solutions flowing in confining pore channels, subject to hydrodynamic forces, and Brownian motion. The numerical simulations are developed by deriving appropriate algorithms based in Jeffery formalism (in 3D) for the particle dynamics. A theoretical model is intensively developed to treat a number of basic ingredients for the simulation algorithms for the pore channels, in particular the dynamic restitution of the nano-particles due to their diffusive collisions at the pore boundaries, the appropriate boundary conditions flow at atomically rough solid boundaries, and the influence of Brownian dynamics in the colloidal suspensions. These and other ingredients for the numerical codes are designed to calculate rigorously and efficiently the PDF distributions for the molecular-particles under equilibrium dynamics. The PDF distributions for the orientations of these particles are calculated by applying the numerical simulations. This procedure also permits calculating the nematic order parameters for the colloidal suspensions on a nano scale in 3D. The calculations of the PDF distributions and nematic order parameters are carried out for diverse species of molecular nano-particles, to investigate the influence of their varying forms from rod-like to ellipsoidal with corresponding diffusion rates.
\end{abstract}

Index Terms-Computer simulation, colloidal suspension particles, ellipsoidal like particles, rod like particles, three dimensional spatial frames.

\section{INTRODUCTION}

The dynamics of molecular polymer particles in dilute colloidal suspensions in solutions flowing inside pores of variable submicron sizes are long-standing research topics that continue to pose outstanding questions. Aside from their importance for traditional applications such as, coatings [1], [2], polymer processing, and catalysis [3], the topic of the flow of such colloidal suspensions in porous media, has recently taken on renewed interest in chemical and biological analysis, for example in protein diffusion in membranes [4] and in the circulation of red blood cells and platelets inside the human body [5], [6]. There is a great amount of research work which has also addressed the problem of the dynamics of colloidal particles suspended in a flowing solution with biological and engineering

Manuscript received December 14, 2013; revised February 8, 2014.

A. Hijaziand A. Atwi are with the Department of Physics, Faculty of Science I, Lebanese University, Hadat-Beirut, Lebanon (e-mail: abhijaz@ul.edu.lb, atwiali-23@hotmail.com: corresponding author).

A. Khater is with the Institut des Molécules et Matériaux du Mans (IMMM, MR6283), Université du Maine,F-72085 Le Mans, France(e-mail: antoine.khater@univ-lemans.fr). applications [7]-[10]. Colloidal particles have a variety of forms, including rod-like and ellipsoidal particles, which show a rich Brownian dynamics. Rigid rod-like and ellipsoidal molecular particles have gained considerable technological importance in the production of high-modulus and high-strength fibers and films due to their excellent mechanical properties [11], [12].

The origin for most current models for the study of the dynamics of colloidal non-spherical particles in a shear flow is historically the approach proposed quite early by Jeffery [13]. This pioneering research work investigated the rotational behavior of a single ellipsoidal particle in a Newtonian fluid. The overall rotation of the colloidal particles in the bulk liquid is referred to as a Jeffery orbit, which will be discussed in detail in Section II.

Our study is focused on the determination of the PDF distributions and the corresponding nematic order parameter for the positions and the orientations of the dilute colloidal suspensions of rod-like and ellipsoidal molecular particle, in the 3D-spatial frame. We have chosen also to calculate, by the available simulation codes, the interactions of these particles with two types of surface boundaries, namely the idealized flat and the rough. This permits to illustrate the difference between the equilibrium dynamics in the depletion layer next to both types of surface boundaries, and to compare their corresponding distributions.

It is well known that it is intractable to study by the only analytical means the dynamics of the molecular polymer particles in dilute colloidal suspensions near solid surfaces, because of the random nature of the Brownian motion and the equally random nature of diffusive collisions. The only viable alternative is to do this by numerical simulations with appropriate algorithms

\section{Colloidal Particle Model in 3D-Spatial Frames}

Particles moving in a suspension tend to orient themselves in the direction of the hydrodynamic flow shearing which can be quantified by fluid velocity gradients. Jeffery [13] showed for a bulk fluid that a single fiber has a closed periodic tumbling motion in simple shear flow, often referred to Jeffery's orbit. Even though this periodic motion has been validated in experiments for dilute bulk suspensions [14]-[16], the interaction of polymer particles with each other as their concentration increases, and with the solid surface boundary of pores, leads to other induced ordinations.

Our present research work deals with dilute suspensions of colloidal particles and hence does not consider the 
interaction effects between the particles; we focus instead on the polymer particle - solid surface boundary interactions.

The orientations of dilute colloidal particles in the bulk of a flowing fluid in a 2D-spatial frame have been studied early by Boeder [17], who introduced a differential equation (BDE)taking into account the dynamic effects due to the Brownian and hydrodynamic forces acting on the particles. In this paper we go beyond the algorithm based in Boeder's BDE and used previously for the 2D-spatial frame [18]-[20], in order to establish an appropriate algorithm based in Jeffrey's equations to calculate the appropriate stochastic PDF distributions in the 3D-spatial frame, for rod-like and ellipsoidal particles in bulk liquid solutions and in the vicinity of the solid surface boundaries of surrounding pores. The mechanical restitution model [18]-[20] at the solid boundaries is extended consequently to $3 \mathrm{D}$, and combined with the detailed implementation of Jeffery's equations not only in the bulk but also in the depletion layer.

We consider in general a simple shear flow acting on an ellipsoidal particle,

The motion of a solid ellipsoid particle, suspended in a simple shear flow is computed analytically [13], neglecting the inertia of the fluid and the particle. The ellipsoidal orientation is defined by three angles $(\theta, \varphi, \psi)$, which determine the Cartesian coordinates $x, y, z$. In contrast, a local coordinate system $x^{\prime}, y^{\prime}, z^{\prime}$, translates and rotates with the ellipsoidal particle, see Fig. 1. In Jeffery's theory it is assumed that the ellipsoidal center translates with the same linear velocity as that of the particle centriod.

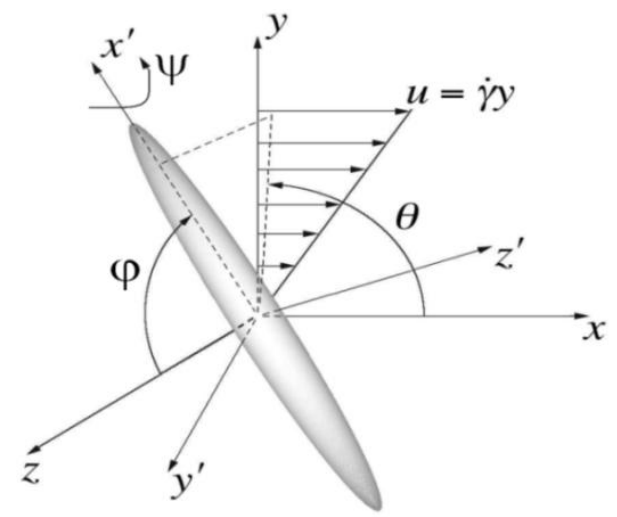

Fig. 1. Coardinate system for ellpsoidal particle centered at the origin.

The angles $\varphi$ and $\theta$ are used to define the unit direction of the primary axis and $\psi$ represents the rotational angle about the ellipsoidal particle. The differential equation governing the time evolution of $\theta$ and $\varphi$ are given by

$$
\begin{gathered}
\dot{\varphi}=\frac{\dot{\gamma}}{4}\left(\frac{r_{\mathrm{e}}^{2}-1}{r_{\mathrm{e}}^{2}+1}\right) \sin 2 \varphi \sin 2 \theta \\
\dot{\theta}=-\dot{\gamma}\left(\frac{1}{r_{\mathrm{e}}^{2}+1}\right)\left(r_{\mathrm{e}}^{2} \sin ^{2} \theta+\cos ^{2} \theta\right)
\end{gathered}
$$

In these equations, $r_{\mathrm{e}}=L / d,(L$ and $d$ are respectively the length and diameter of the macromolecular particle), is the aspect ratio of the ellipsoidal particles, $\theta$ is defined as the angle of the particle with respect to flow direction, $\varphi$ as the vorticity axis; $\varphi=\frac{\pi}{2}$ when the ellipsoidal particle lies in the plane of shear.
Note that for large $r_{\mathrm{e}}>>1,(L>>d)$, the ellipsoidal form turns into rod-like particles, and Jeffery's equations become

$$
\begin{gathered}
\dot{\varphi}=\frac{\dot{\gamma}}{4}\left(\frac{r_{e}^{2}-1}{r_{e}^{2}+1}\right) \sin 2 \varphi \sin 2 \theta \cong \frac{\dot{\gamma}}{4} \sin 2 \varphi \sin 2 \theta \\
\dot{\theta}=-\dot{\gamma}\left(\frac{r_{e}^{2}}{r_{e}^{2}+1}\right) \sin ^{2} \theta-\dot{\gamma}\left(\frac{1}{r_{e}^{2}+1}\right) \cos ^{2} \theta \cong-\dot{\gamma} \sin ^{2} \varphi
\end{gathered}
$$

The $\theta$ and $\varphi$ defined in a fixed Cartesian coordinate system, as shown in Fig. $(-\pi / 2 \leq \theta \leq+\pi / 2$ and $0 \leq \varphi \leq \pi)$, are sufficient because the problem is invariant under the transformation $\varphi \rightarrow \varphi+\pi$.

The hydrodynamic force tends to align the particles in the direction of the shear flow, $\theta$ is taken as positive or negative in the trigonometric sense. To simulate the hydrodynamic effects in a time interval $\Delta t$ between two successive simulation events labelled $s$ and $s+1$, we compute $\Delta \theta_{\text {hyd }}(s+1 ; s)$, and $\Delta \varphi_{\text {rot }}(s+1 ; s)$, the hydrodynamic rotation about the centre of mass of the particles, using the following algorithm

$$
\Delta \varphi_{\text {hyd }}(s+1 ; s)=\varphi\left(t_{s+1}\right)-\varphi\left(t_{s}\right) \cong \frac{\gamma^{\prime}}{4} \sin 2 \varphi \sin 2 \theta \Delta t
$$

$$
\Delta \theta_{h y d}(s+1 ; s)=\theta\left(t_{s+1}\right)-\theta\left(t_{s}\right) \cong-\gamma^{\prime} \sin ^{2} \theta \Delta t
$$

In contrast the Brownian forces in the liquid solution create a diffusive rotational motion of the particles, for which the rotation variables $\Delta \theta_{\text {rot }}(s+1 ; s)$ and $\Delta \varphi_{\text {rot }}(s+$ $1 ; s)$ can then be assumed in the algorithm as previously for $\Delta \theta_{\text {rot }}$ in the form

$$
\begin{aligned}
& \Delta \varphi_{\text {rot }}(s+1 ; s)= \pm \Delta \varphi_{\text {rot }} \\
& \Delta \theta_{\text {rot }}(s+1 ; s)= \pm \Delta \theta_{\text {rot }}
\end{aligned}
$$

The Brownian rotations, $\pm \Delta \varphi_{\text {rot }}$ and $\pm \Delta \theta_{\text {rot }}$, are clock and anticlockwise. In this procedure the simulation time interval $\Delta t$ is related, as previously [18]-[20], to an effective variable for the Brownian rotation diffusion by the following equations

$$
\begin{aligned}
\Delta \varphi_{\text {rot }}^{2} & =2 D_{\varphi r o t} \Delta t \\
\Delta \theta_{\text {rot }}^{2} & =2 D_{\theta \text { rot }} \Delta t
\end{aligned}
$$

$D_{r o t}$ is the rotational diffusion coefficient of the macromolecular particle about its centre of mass. For the small $\Delta t$ simulation time intervals to be unique, at least for hydrodynamic events, they must satisfy

$$
\Delta t=\frac{1}{2 D_{\theta \text { rot }}} \Delta \theta_{\text {rot }}^{2}=\frac{1}{2 D_{\varphi r o t}} \Delta \varphi_{r o t}^{2}
$$

This leads to the simulation relationship

$$
\Delta \theta_{\text {rot }}^{2}=\frac{2 D_{\theta \text { rot }}}{2 D_{\varphi r o t}} \Delta \varphi_{\text {rot }}^{2} \equiv \mathrm{r}_{\theta, \varphi} \Delta \varphi_{\text {rot }}^{2}
$$


There are two ways to treat $r_{\theta, \varphi}$ in the numerical simulations, as follows

$$
\begin{aligned}
& \mathrm{r}_{\theta, \varphi}=\frac{D_{\theta r o t}}{D_{\varphi r o t}}=1 \\
& \mathrm{r}_{\theta, \varphi}=(\sin \varphi)^{-2}
\end{aligned}
$$

Eq.(8a) is based on the assumption of independent and random Brownian simulation events, where the rod-like particle symmetry cuts the same diffusion coefficient in whatever angular displacement it makes. This underestimates somewhat the physical relation of $\Delta \theta_{\text {rot }}$ and $\Delta \varphi_{\text {rot }}$ for hydrodynamic events, which can be derived directly as in (8b). However, Eq.8b would overestimate this relationship in the global simulation since random Brownian events may be viewed as disconnected. Eqs.(8a) and (8b) yield respectively

$$
\begin{gathered}
\Delta \theta_{\text {rot }}(s+1 ; s)= \pm \Delta \theta_{\text {rot }} \\
\Delta \varphi_{\text {rot }}(s+1 ; s)= \pm \sin \varphi \Delta \theta_{\text {rot }}
\end{gathered}
$$

We have used both Eq. (9a) and Eq. (9b) to run our overall simulations, for a variety of hydrodynamic conditions. The results do not differ in any significant manner. It is noticeable however that we gain on the calculation time when simplifying the simulation conditions, by considering $r_{\theta, \varphi}=1$.

The time interval $\Delta \mathrm{t}$ can be now eliminated from the hydrodynamic algorithm using Eq. (8a), so that equations Eq. (3a) and (3b) become

$$
\begin{gathered}
\Delta \theta_{h y d}=-\frac{1}{2} \alpha \sin ^{2} \theta \Delta \varphi_{r o t}^{2} \\
\Delta \varphi_{h y d}=\frac{1}{8} \alpha(\sin 2 \theta \sin 2 \varphi) \Delta \varphi_{r o t}^{2}
\end{gathered}
$$

\section{Simulation Results For Pore Channels}

The simulation results are presented for the bulk and the depletion layer over the interval $0 \leq \zeta=\mathrm{z} / \mathrm{L} \leq 0.5$ next to the solid surface boundary. The simulations in the depletion layer are made for atomically flat boundaries and for Gaussian rough surface boundaries. $\zeta=0$ is the reference plane corresponding to the lowest material step levels that a particle extremity can touch in diffusive collisions.

\section{A. PDF Distribution Results for Rod-Like Particles in the Bulk Solution}

Fig. 2a, and Fig. 2b, present the normalized PDF orientation distributions, $P(\theta, \varphi)$, as a 3D contour fill surface, over the plane $(\theta, \varphi)$, for rod-like macromolecular particle in the bulk of a flowing solution in the 3D-spatial frames, for different Peclet numbers $\alpha$, from a relatively low value, as in Fig. 2a, to a high value Fig. 2b. The angles $\theta$ and $\varphi$ represent the orientational angle in $3 \mathrm{D}$ space for the rod-like particles. Note that we use a color scheme in these figures: red corresponds to the highest probability for the PDF distributions, and black to the lowest. Note also that the absolute numerical values per color interval may vary from one set of PDF figures to another.

The colloidal rod-like particles in dilute suspensions orient themselves in the bulk of the flowing solution under the influence of two primary forces, the Brownian and the hydrodynamic forces. The hydrodynamic force tends to act on the particle to turn it in the shear flow $\left(\theta=0^{\circ}\right)$. In general, the simulation results, valid for the open interval

$$
\varphi=] 0^{\circ}, 180^{\circ}[
$$

Indicate that for low flow conditions, with a typically low Peclet number $\alpha=1$, the Brownian effect is dominant for the rod-like particles in comparison with the hydrodynamic motion. For high flow conditions, which correspond to typically high Peclet numbers, $\alpha 100$, the influence of Brownian motion is less important. For the higher Peclet flow the particle orients increasingly in the direction of the flow under the dynamic equilibrium conditions. Moreover, the PDF probability around $\theta=0$ and $\varphi=\pi / 2$ increases with increasing $\alpha$.

The above results are calculated by numerical simulations. However, we do dispose at present of any experimental data to compare with, in particular for the nematic order parameter in the neighborhood of solid surface boundaries in the depletion layer.
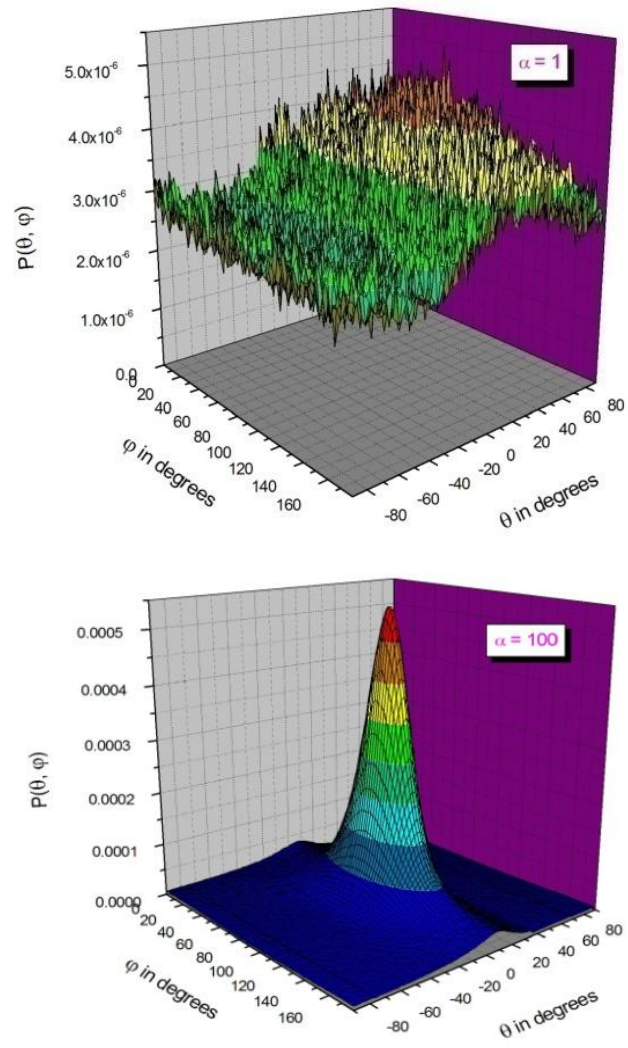

Fig. 2. Simulation results for normalized angular $\operatorname{PDF}, P(\theta, \varphi)$, in the bulk for Peclet number a) $\alpha=1$ and b) $\alpha=100$.

The PDF distribution for $\alpha=1$, Fig. 2a, shows a broad and symmetrical distribution about the vorticity axes $\varphi=\pi / 2$, with appreciable suspension concentration in the orientation solid angle frame $\left\{\theta \approx\left[10^{\circ}, 50^{\circ}\right], \approx\left[60^{\circ}\right.\right.$, $\left.\left.120^{\circ}\right]\right\}$. The existence of this broad distribution in the $(\theta, \varphi)$ plane comes from the Brownian motion. Increasing the 
hydrodynamic flow to Peclet numbers $\alpha=100$, increases the tendency of the colloidal particles to regroup in significant concentrations, under equilibrium dynamics, in the narrow solid angle frames $\left\{\theta \approx\left[5^{\circ}, 10^{\circ}\right], \approx\left[85^{\circ}, 95^{\circ}\right]\right\}$ for $\alpha=$ 100, as in Fig. $2 b$.

\section{B. PDF Distribution Results for Ellipsoidal -Like Particles in the Bulk Solution with Different Aspect Ratio}

Fig. 3a, and Fig. 3b, and Fig. 4a, and b present the angular normalized PDF distributions, $P(\theta, \varphi)$, as a 3D contour fill surface, over the plane $(\theta, \varphi)$, for ellipsoidal macromolecular particle in the bulk solution, for different Peclet numbers $\alpha$. The results correspond to gradually increasing the Peclet number from a relatively low value, as in Fig. 3a, and $b$ to a high value in Fig. 4a, and Fig. 4b. This is done in each case for the aspect ratios: $r_{e}=1.02$ and 2 which correspond in each group respectively to $a$, and $b$. the angles $\theta$ and $\varphi$ represent the orientation angle for the ellipsoidal particles in the 3D-spatial frame. Note that we use a color scheme in these figures, where red corresponds to the highest probability for the PDF distributions, and black to the lowest. Note also that their absolute numerical values need not necessarily have the same value from one set of figures to another.
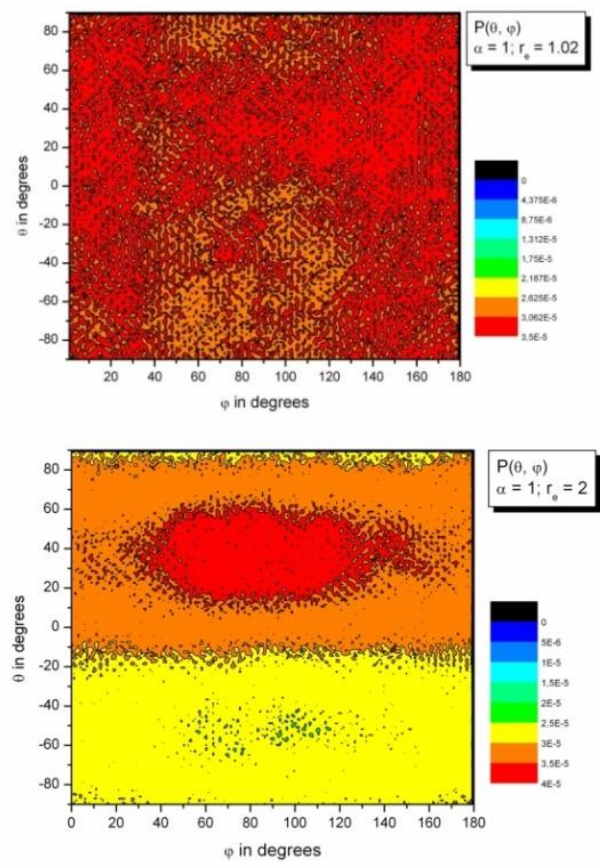

Fig. 3. Simulation results for normalized angular PDF, $P(\theta, \varphi)$, in the bulk at law Peclet number $\alpha=1$ a): $r_{e}=1.02$; (b) $: r_{e}=2$.

In general, the simulation results, valid for the open interval

$$
\varphi=] 0^{\circ}, 180^{\circ}[
$$

Indicate that for low flow conditions with a typically low Peclet number $\alpha=1$, the Brownian effect is dominant for the particles in comparison with the hydrodynamic motion. For relatively medium and high flow, which correspond to typically high Peclet numbers $\alpha=100$, the influence of Brownian motion is less important compared to that due to the hydrodynamic motion. For the higher Peclet flow the macromolecular particle orients increasingly in the direction of the flow under the dynamic equilibrium conditions.
Moreover, and regardless of the value of the aspect ratio value, the PDF probability around $\theta=0$ and $\varphi=\pi / 2$ increases with increasing $\alpha$.

Fig. 3a and Fig. 3b, present the simulation results for the normalized PDF distributions of the colloidal particles in the bulk solution, under the conditions of dynamic equilibrium, as a function of a fixed small Peclet number, $\alpha=1$, with increasing aspect ratios $r_{e}=1.02$, and 2 . The nearly spherical particles for aspect ratio $r_{e}=1.02$, yield the results presented in Fig. 3a. These indicate, as expected, a relatively uniform distribution in the $(\theta, \varphi)$ plane, with already some perceptible symmetry about the $\varphi=\pi / 2$ direction. We find this symmetry for all the results of the numerical simulations since the Brownian motion dictates this. To understand Fig. $3 \mathrm{a}$ it is important to recall that a particle with spherical symmetry, for which the aspect ratio is strictly $r_{e}=1$, has no preferential axis with respect to the flow direction or with respect to the vorticity axes. For such a perfect symmetry we expect that the PDF would be perfectly uniform in the $(\theta, \varphi)$ plane. Fig. $3 b$ shows next the normalized PDF distributions for the greater $r_{e}=2$ aspect ratio. In this case the PDF distribution is visibly different from that for $r_{e}=$ 1.02, for which the colloidal suspension acquires an orientation tendency, with a distribution that presents particle concentrations in the narrower solid angle window \{ $\left.\approx\left[10^{\circ}, 40^{\circ}\right], \varphi \approx\left[30^{\circ}, 150^{\circ}\right]\right\}$.
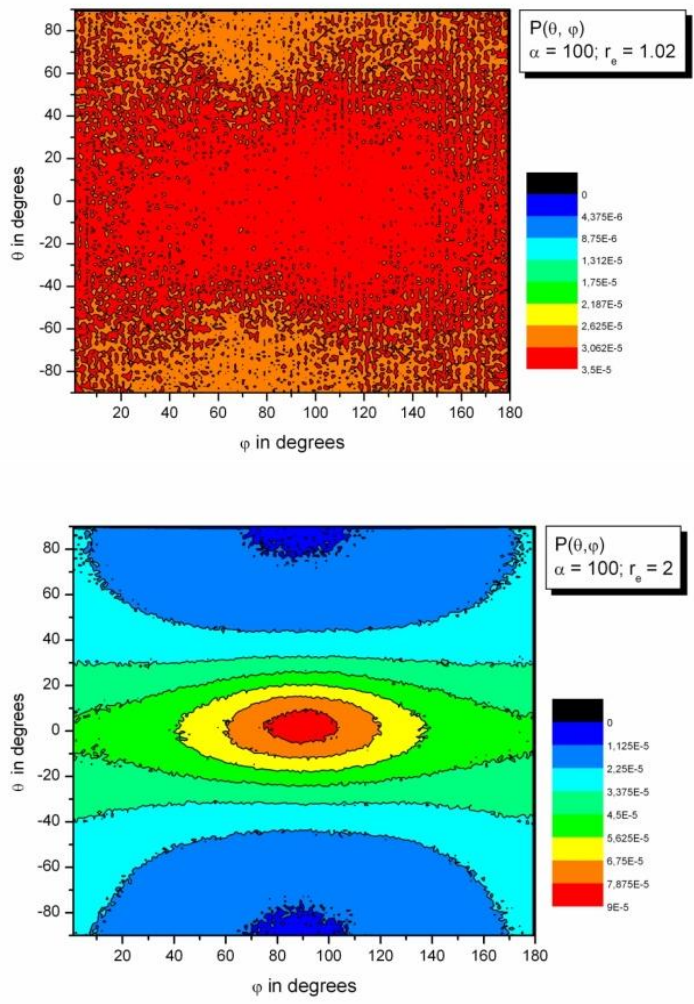

Fig. 4. Simulation results for normalized angular PDF, $P(\theta, \varphi)$, in the bulk at high Peclet number $\alpha=100 \mathrm{a}): r_{e}=1.02$; (b): $r_{e}=2$

Comparing the PDF results of Fig. 3a, and Fig. 4a, it is evident that increasing the Peclet number $\alpha$ for the fixed aspect ratio, $r_{e}=1.02$, does not change the overall aspect of the normalized PDF distributions. Increasing the strength of the hydrodynamics flow increases, however, the concentration of the particles in the direction of flow and reduces the importance of the contribution of the Brownian 
motion.

Comparison of Fig. 3b, and Fig. 4b, shows next the simulation results for colloidal suspensions with aspect ratio $r_{e}=2$, for the increasing Peclet number, $\alpha=1$, and 100. It is again observed that increasing the flow will accentuate the PDF distributions into smaller windows in the $(\theta, \varphi)$ plane. In contrast with the calculated effects at small $\alpha$, the results for high hydrodynamic flow conditions show that the colloidal particles tend to regroup in significant concentrations, under the conditions of dynamic equilibrium, in the narrow solid angle window

\section{NEMATIC ORDER TENSOR S}

The nematic phase is characterized by long-range orientation order; i. e. the long axis of the ellipsoidal molecules tends to align along a preferred direction. The locally preferred direction may vary throughout the medium.

Given the complexity of the colloidal suspension, it is necessary to consider a tensor description of their order which commonly called as the nematic phase order in the solution. A natural order parameter to describe the ordering, is the second rank tensor [21], as follows

$$
\mathrm{S}_{\alpha \beta}(r)=\frac{1}{N} \sum_{i}\left(u_{\alpha}^{(i)} u_{\beta}^{(i)}-\frac{1}{3} \delta_{\alpha \beta}\right)
$$

$N$ is the number of el particles in the solution and the indices correspond to Cartesian directions $\alpha, \beta=(x, y, z)$, and $\delta_{\alpha \beta}$ is second rank unit tensor

$$
\delta_{\alpha \beta}=\left\{\begin{array}{l}
1 \text { if } \alpha=\beta \\
0 \text { if } \alpha \neq \beta
\end{array}\right.
$$

In our system coordinate system

$$
u_{x}=\sin \varphi \cos \theta ; u_{y}=\sin \varphi \sin \theta ; u_{z}=\cos \varphi,
$$

These yields to

$S=$

$\int_{\frac{-\pi}{2}}^{\frac{\pi}{2}} \int_{0}^{\pi}\left(\begin{array}{ccc}\sin ^{2} \varphi \cos ^{2} \theta & \sin ^{2} \varphi \cos \varphi \sin \theta & \sin \varphi \cos \varphi \cos \theta \\ \sin ^{2} \varphi \cos \varphi \sin \theta & \sin ^{2} \varphi \sin ^{2} \theta & \sin \varphi \cos \varphi \sin \theta \\ \sin \varphi \cos \varphi \cos \theta & \sin \varphi \cos \varphi \sin \theta & \cos ^{2} \varphi\end{array}\right) \sin \varphi P(\theta, \varphi) d \varphi d \theta$

As previously, $P(\theta, \varphi)$ is the probability to find the ellipsoidal particles with the orientation given by the angles $\theta$ and $\varphi$, i.e. the PDF in the 3D-spatial frame. This tensor form has been used extensively in the molecular theory for colloidal particles [22], for fiber-like charged particles [23], and in recent research studies [24], [25].

\section{Calculation of the Nematic Order Tensor for Bulk Solution}

In this part the order parameter tensor $S_{\alpha, r_{e}}$ for ellipsoidal particles is calculated for different aspect ratios $r_{e}$, and Peclet numbers $\alpha$. The sense of the indices $\left(r_{e}, \alpha\right)$ on $\mathrm{S}$ are evident.

We give next, in matrix form following the Cartesian directions, the numerical results for the calculated $S_{\alpha, r_{e}}$ in bulk solution for $\left(r_{e}, \alpha\right)=(1,1.02),(100,2)$, using the corresponding normalized PDF distributions $P(\theta, \varphi)$ calculated previously in Section III and Section IV.

$$
\begin{gathered}
S_{1,1.02}\left(\begin{array}{ccc}
0.422 & 0 & 0 \\
0 & 0.409 & 0 \\
0 & 0 & 0.201
\end{array}\right) \\
S_{100,2}\left(\begin{array}{ccc}
0.609 & 0 & 0 \\
0 & 0.243 & 0 \\
0 & 0 & 0.204
\end{array}\right)
\end{gathered}
$$

It should be clear from the above that $S_{\alpha, r_{e}}$ varies with $\left(r_{e}, \alpha\right)$.

The first matrix is for quasi-spherical particles with a small aspect ratio $r_{e}=1.02$. for low Peclet numbers, $\alpha=1$, the results are not significantly modified despite the two orders of magnitude increase in the hydrodynamic flow. It is also noticeable that the preferred alignment in this case is along the $X$ and $Y$ directions in the plane of the flow, as in Fig. 5, with a weaker component alignment along the $Z$ direction normal to the flow. Increasing the aspect ratio $r_{e}$ from 1.02 to 2, under hight flow conditions is sufficient to introduce new effects. The favored alignment is visibly in the $X$ direction with weaker component alignments along the $Y$ and $Z$ directions.

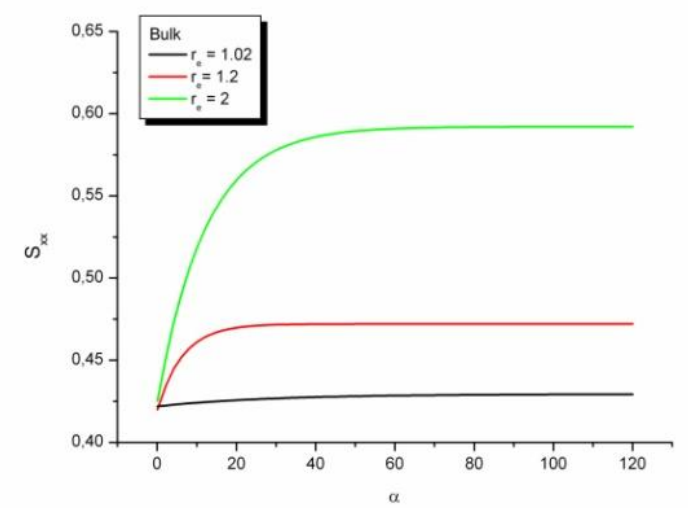

Fig. 5. Nematic order parameter $S_{x x}$ in X-direction as a function of Peclet number $\alpha$ for different ellipsoidal particles in bulk.

\section{CONCLUSIONS}

We have developed algorithms and carried out numerical simulations to analyze the dynamics of dilute colloidal suspensions of macromolecular particles in solutions flowing open channel pore. The pores are modeled in a three-dimensional frame of reference with boundaries. The colloidal particles are subject to hydrodynamic forces and Brownian motion in bulk solution. The numerical simulations are carried out to calculate in particular the spatial statistical PDF distributions for the spatial the orientations of ellipsoidal and rod like particles in colloidal suspensions in a solution under equilibrium dynamics for the bulk liquid. The simulations are developed for and open pore channels, and are valid throughout the space of the pores and in the boundary depletion layers, for a wide variety of hydrodynamic flow conditions, at low, intermediate, and high flow, characterised by the rotational Peclet number and for variable aspect ratios characteristic of the ellipsoidal particles under study.

Our simulations yield directly the nematic order parameter for colloidal suspensions in the over its tensorial 
representation, for a variety of forms of ellipsoidal particles selected to correspond to real molecules particles.

\section{ACKNOWLEDGMENT}

This work was supported in part by the Lebanese University Grant

\section{REFERENCES}

[1] M. Fuji, H. Fujimori, T. Takei, T. Watanabe, and M. Chikazawa, "Motion of inertial spheroidal particles in a shear flow near a solic wall with special application to aerosol transport in microgravity," The Journal of Physical Chemistry B, vol. 102, pp. 10498-10504, 1998.

[2] B. P. Binks, "Particles as surfactants—similarities and differences," Current Opinion in Colloid \& Interface Science, vol. 7, pp. 21-41, 2002.

[3] H. Jia, G. Zhu, and P. Wang, "Catalytic behaviors of enzymes attached to nanoparticles: The effect of particle mobility," Journal of Biotechnology and Bioengineering, vol. 84, pp. 406-414, 2003.

[4] A Sokolov, I. Aranson, J. Kessler, and R. Goldstein, "Concentration dependence of the collective dynamics of swimming bacteria," Physical Review Letters, vol. 98, p. 158102, 2007.

[5] C. Caro, T. Pedley, R. Schroter, and W. Seed, "The Mechanics of the circulation,"Oxford University Press, 1978.

[6] H. L. Goldsmith, "The flow of model particles and blood cells and its relation to thrombogenesis progress in Hemostasis and Thrombosis," Grune and Stratton Inc., New York, NY, 1972.

[7] J. Park, B. Metzger, É. Guazzelli, and J. E. Butler, " A cloud of rogid fibers sedimenting in a viscous fluid," Journal of Fluid Mechanics, vol. 648 , pp. 351-362, 2010.

[8] K. A. Rose, B. Hoffman, D. Saintillan, E. S. G. Shaqfeh, and J. G. Santiago, "Hydrodynamic interactions in metal rodlike-particle suspensions due to induced charge electroosmosis," Physical Review E, vol. 79, p. 011402, 2009.

[9] G. Subramanian and D. L. Koch, "Inertial effects on fibre motion in simple shear flow," Journal of Fluid Mechanics, vol. 535, pp. $383-$ $414,2005$.

[10] Y. Zhang, A. Done, T. Weisgraber, B. Alder, M. Graham, and J. J. de Pablo, "Tethered DNA dynamics in shear flow," Journal of Chemical Physics, vol. 130, p. 13, 2009.

[11] W. W. Adams, R. K. Eby, and D. E. McLemore, "The materials science and engineering of rigid-rod polymers materials research society symposia proceedings," Pittsburgh, PA, 1989.

[12] J. Gustin, A. Joneson, M. Mahinfalah, and J. Stone, "Low velocity impact of combination Kevlar/carbon fiber sandwich composites," Composite structures Jounral, vol. 69, pp. 396-406, 2005.

[13] G. B. Jeffery, "The motion of ellipsoidal particles immersed in a viscous fluid," Proceedings of the Royal Society of London. Series A, vol. 102, pp. 161-179, 1922.

[14] S. G. Mason and R. S. J. Manley, "Particle motions in sheared suspensions: Orientation and interactions of rigid rods," Proceedings of the Royal Society of London. Series A, vol. 238, pp. 117-131, 1956.

[15] G. Taylor, "The motion of ellipsoidal particles in a viscous fluid," Proceedings of the Royal Society of London. Series A, vol. 103, pp. 58-66, 1923

[16] B. J. Trevelyan and S. G. Mason, "Particle motions in sheared suspensions. I. Rotations," Journal of Colloid Science, vol. 6, pp. 354 367, 1951.

[17] P. Boeder, "Über Strömungsdoppelbrechung," Zeitschrift für Physik A Hadrons and Nuclei, vol. 75, pp. 258, 1932.

[18] A. Hijazi and A. Khater, "Boëder PDF Brownian simulations for macromolecular rod-like particles near uneven solid surfaces," European Polymer Journal, vol. 44, pp. 3409, 2008.
[19] A. Hijazi and A. Khater, "Brownian dynamics simulations of rigid rod-like macromolecular particles flowing in bounded channels," Computational Materials Science, vol. 22, pp. 279, 2001.

[20] A. Hijazi and A. Khater, "Simulations of distribution functions for rod-like macromolecules in linear flow near solid surfaces," Computational Materials Science, vol. 20, pp. 213, 2001.

[21] P. Oswald and P. Pieranski, "Nematic and cholesteric liquid crystals," CRC Press, Taylor \& Francis Group, United States of America, 2005.

[22] M. Doi and S. F. Edwards, "Faraday transactions 2: Molecular and chemical physics," Journal of the Chemical Society, vol. 74, pp. 560$570,1978$.

[23] S. Advani, "The use of tensors to describe and predict fiber orientation in short fiber composites," Journal of Rheology, vol. 31 pp. 751, 1987.

[24] A. Redjeb, École Nationale Supérieure des Mines de Paris, École Doctorale 364 : Sciences Fondamentales et Appliquées 2007, pp. 244.

[25] G. Ausias, Laboratoire de Génie Mécanique et Matériaux, Université de Bretagne-Sud 2007, pp. 140.

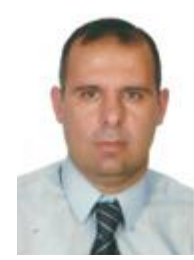

Abbas Hijazi is a full professor at the Physics and Electronics Department of the Lebanese University, since 2003. He serves currently as the coordinator of the Physics Master M2 program. He received his B.Sc. degree in Industrial Computer Sciences in 1992 from the ISTG, Grenoble University, France, and his Ph.D. in Physics in 2000 from the University du Maine, Le Mans, France. He worked during 2000-2002 as Lecturer at the University du Maine. His research interests are in numerical simulations, Molecular Dynamics, and wireless sensor network.

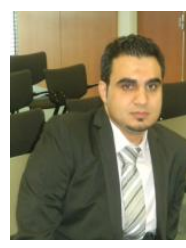

Ali Atwi is a part time lecturer at the Lebanese University. He received his B.Sc. Physics degree in 2007 from the Lebanese University, Beirut, Lebanon. He received his M.Sc. physics degree in 2008 and Ph.D physics degree in 2012 at the University du Maine, Le Mans, France. His research interests are in the dynamics, mobility and statistical physics of colloidal suspensions of molecular and nano particles in confined porous systems, and their biophysical applications.

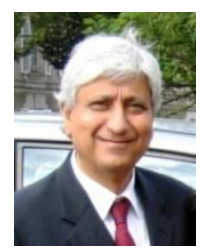

Antoine Khater is a university distinguished professor (CE) at the University du Maine, Le Mans, France. He received his B.Sc. theoretical physics degree with Honors 1968, and his Ph.D. physics degree 1972, from the University of Essex, Colchester, UK, working with professor R Loudon (FRS). He is presently a Team Leader Nanomagnetism and Modeling, and the Head of the Computational Materials Physics group, at the Institute for Molecules and Materials UMR 6283 CNRS, at the University du Maine, France. He has been the director of the Laboratory for Materials Science URA 807 CNRS 1989-2005, the dean of the Graduate School of Science 1993-1999 and a member elect of the Board of Governors 19961999, of the University du Maine, France. A member elect of the board of Governors 1995-1998 of the French Physical Society and president of its Committee for Mediterranean Physics 1997-2003. He was the director of the Raman Light Scattering Laboratory at the Lebanese University, Beirut, Lebanon in 1974-1976. His research interests are in theoretical and numerical methods, and numerical simulations, dynamics of colloidal suspensions in confined liquids, nanomagnetism and magnetism of materials, transport of electrons, magnons and phonons in ordered and disordered nanostructures, surface and interfaces physics, and light scattering and absorption in materials science. 